\title{
Total and subtotal replacement of the common bile duct: effect of transhepatic silicone tube stenting
}

\author{
A CUSHIERI, P R BAKER, R J L ANDERSON, AND M P HOLLEY \\ From the Departments of Surgery and Pathology, Ninewells Hospital and Medical School, Dundee
}

SUMmaRY The effect of silicone tube stenting on the outcome of total and subtotal replacement of the bile duct with autologous vein was investigated in experiments performed in pigs. All but one of the unstented animals developed dense strictures of the extrahepatic ductal system. This complication was abolished by the use of silicone stents passed transhepatically. The vein autografts in the stented animals underwent progressive shortening with elongation of the distal common bile duct so that beyond four months only one suture line was encountered, and no recognisable venous element was detectable on histological examination. Apart from minor dilatation of the intrahepatic biliary tree encountered in some animals, all the surviving stented animals progressed well and had normal serial liver function tests.

\begin{abstract}
Although there seems to be good evidence of regneration of bile duct epithelium, the literature regarding the outcome of various bile duct grafts in large animals is conflcting. Vein autografts, arterial homografts, ureter and synthetic grafts have been used. ${ }^{-9}$ The procedures used in these experiments have varied from division of the bile duct with interposition of a tubular graft, subtotal excision of the circumference of the bile duct with patch grafting, to total excision of varying lengths of bile duct or strictures and replacement by biological or synthetic grafts. In some of the experimental work with biological grafts, a tendency to stenosis with proximal bile duct dilatation and impaired biliary drainage has been reported. ${ }^{37}$ In all these experiments, however, the precise effect of stenting of the grafts was not investigated. The purpose of the present study was to determine the effect of stenting on the outcome after both total and subtotal replacement of the bile duct with autologous vein.
\end{abstract}

\section{Methods}

All the experiments were performed in young pigs (Landrace/Large White cross, $25-30 \mathrm{~kg}$ ). Total or subtotal replacement of the common bile duct was

Address for correspondence: Professor A Cushieri, Department of Surgery, Ninewells Hospital and Medical School, Dundee DD1 9SY.

Received for publication 13 October 1982 with autologous vein (external jugular) which was dissected out through a vertical cervical incision at the start of the procedure.

The operations were carried out under general anaesthesia with endotracheal intubation using halothane, nitrous oxide, and oxygen. Four experimental groups were included: Group I - total replacement without stenting; group II - total replacement with stenting; group III - subtotal replacement without stenting; group IV - subtotal replacement with stenting.

The animals were investigated preoperatively and at varying intervals after surgery using the following tests: liver function tests (bilirubin, serum proteins, alkaline phosphatase, alanine aminotransferase, and $\gamma$-glutamyl transpeptidase), compartmental BSP test and contrast biliary radiology. The animals were killed at varying intervals after surgery and subjected to a detailed postmortem examination with histological examination of the liver and the entire extrahepatic biliary tract.

\section{GROUP I}

Total replacement of the bile duct without stenting. In eight pigs the entire common bile duct just distal to the cystic duct entry was resected down to its entry into the duodenum. It was replaced by a tubular graft of autologous external jugular vein without stenting. The average length of the graft was $3.0 \mathrm{~cm}$. The anastomoses were performed using $5 / 0$ prolene sutures (Fig. 1). 


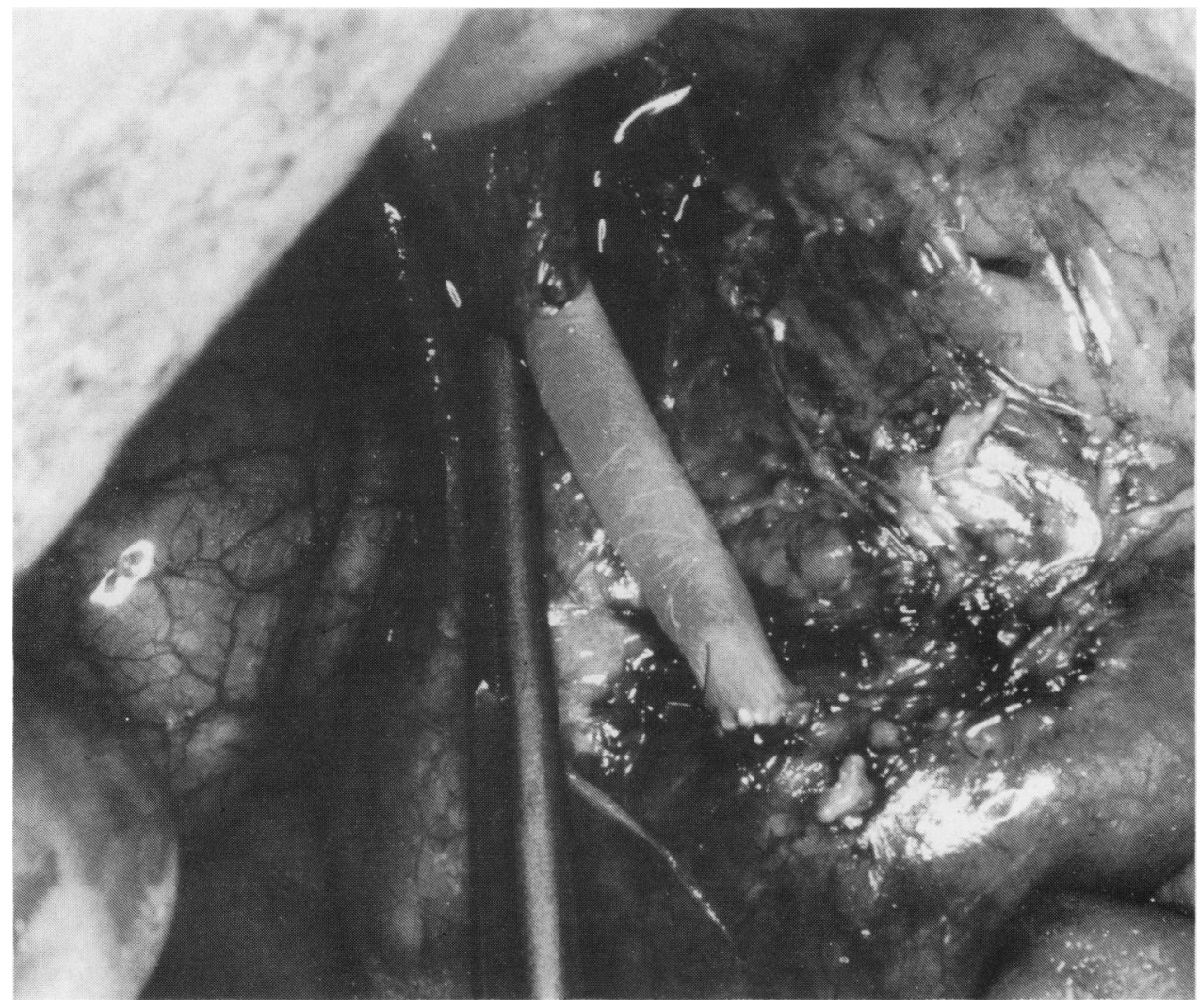

Fig. 1 Total replacement of bile duct from level just distal to cystic duct to duodenum. Gall bladder is left in situ.

GROUP II

Total replacement with stenting. In a further nine animals the same procedure was performed but in addition a transhepatic silicone stent was inserted and left indwelling for periods up to 16 weeks. The intrahepatic portion of the stent had multiple side holes cut out (Fig. 2).

\section{GROUP III}

Subtotal replacement without stenting. In this group of five animals the anterior two thirds of the circumference of the bile duct from the level of the cystic duct to the duodenum was excised leaving an intact $2 \cdot 0-3 \cdot 0 \mathrm{~mm}$ strip joining two ends posteriorly. The autologous vein graft was split longitudinally and sutured as a patch graft using $5 / 0$ prolene sutures.

\section{GROUP IV}

Subtotal replacement with stenting. In a further five pigs, the same procedure as in group III was performed but in addition a transhepatic silicone stent was inserted and left indwelling for periods of up to 12 weeks.

\section{Results}

\section{OUTCOME}

There were five peri-operative deaths (within seven days of the operation) - three from cholangitis with liver abscess formation and two from biliary peritonitis owing to leakage from the anastomatic suture line. Deaths from cholangitis and liver abscess occurred in pigs with indwelling stents and was attributed to aspiration of the stent by the animal technician. This resulted in infection of the hepatobiliary tree. This complication was abolished by daily irrigation of the stent, avoidance of aspiration and by systemic ampicillin therapy for the first 10 days after operation.

In all 22 pigs survived the procedure, 19 were killed at various intervals varying from two to 12 months after surgery. Three animals were kept as long term survivors (12 to 24 months). 


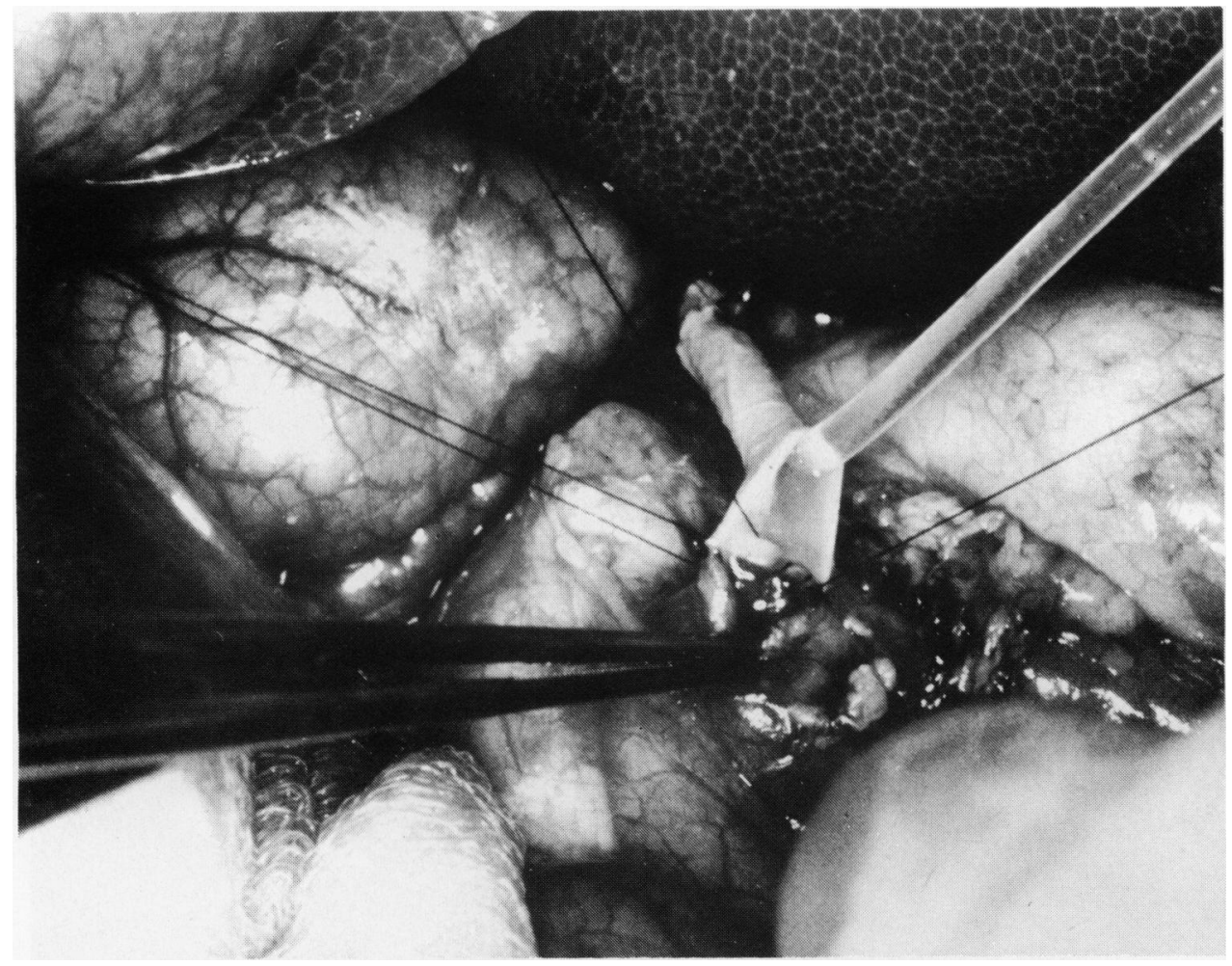

Fig. 2 Total replacement of bile duct with transhepatic silicone tube stenting. Stent had multiple side holes corresponding to its intrahepatic course.

GROUP I

All but one of these animals became jaundiced with biochemical evidence of cholestasis and an impairment of the excretory component of the BSP clearance curve. Seven animals were killed within four months of their operation and a tight concentric bile duct stricture with gross dilitation of the proximal biliary tract was found. The graft was not recognisable macroscopically. The dilated proximal biliary tract contained calcium bilirubinate sludge and soft concretions of this bile pigment. One of the animals in this group progressed satisfactorily with serial normal liver function and compartmental BSP tests. It was kept as a long term survivor. A normal extrahepatic biliary system was found at death two years after surgery.

\section{GROUP II}

Two animals in this group died postoperatively from cholangitis and liver abscess formation. The remainder progressed satisfactorily with weight gain and normal liver function on serial testing. Six of these animals were killed at intervals ranging from eight weeks to nine months. By eight to 12 weeks the vein graft had shortened to $0.5-0.7 \mathrm{~cm}$ and by four months the two suture lines had approximated to each other. Stenosis of the extrahepatic conduit was not encountered in this group. The gross appearance of the common bile duct after 12 months was undistinguishable from normal.

\section{GROUP III}

There were two deaths from biliary peritonitis in this group. The remaining animals developed progressive cholestasis and were found to have marked stenosis of the bile duct at the time of death with gross dilatation of the intrahepatic biliary tree and calcium bilirubinate sludge in the bile.

\section{GROUP IV}

One of these animals died of cholangitis and liver abscess. The remaining four animals did well with normal serial liver function tests and weight gain. The vein patch was shown to have reduced in length to $0.6 \mathrm{~cm}$ by eight to 12 weeks and was macroscopically unrecognisable beyond nine months. 
PATHOLOGICAL FINDINGS

All but one of the surviving pigs in groups I and III developed stricture, histological examination showing dense fibrosis with no recognisable vein graft tissue and proximal severe cholangitis. The hepatic parenchyma showed evidence of marked extrahepatic obstruction with bile duct proliferation, bile plugs, and polymorphonuclear infiltrates in the portal tracts.

In the stented animals from group II, the histological changes on serial sectioning of the reconstituted extrahepatic biliary tract consisted of two components: (1) Progressive shortening (contraction) of the grafted area from an initial length of
$30.0 \mathrm{~mm}$ to $5 \cdot 0-7.0 \mathrm{~mm}$ at eight to 12 weeks with approximation of the distal suture line to the proximal one such that at four months only one suture line was observed just below the level of the cystic duct. This process was accompanied by gradual lengthening of the distal common bile duct. (2) Within four weeks of the operation, the intima of the contracting vein graft was entirely replaced by columnar epithelium similar to that of the intact common bile duct. There was a progressive contraction and disappearance of the vein graft so that by four to six months there was no recognisable venous element in the extrahepatic bile duct system on serial histological examination (Fig. 3).
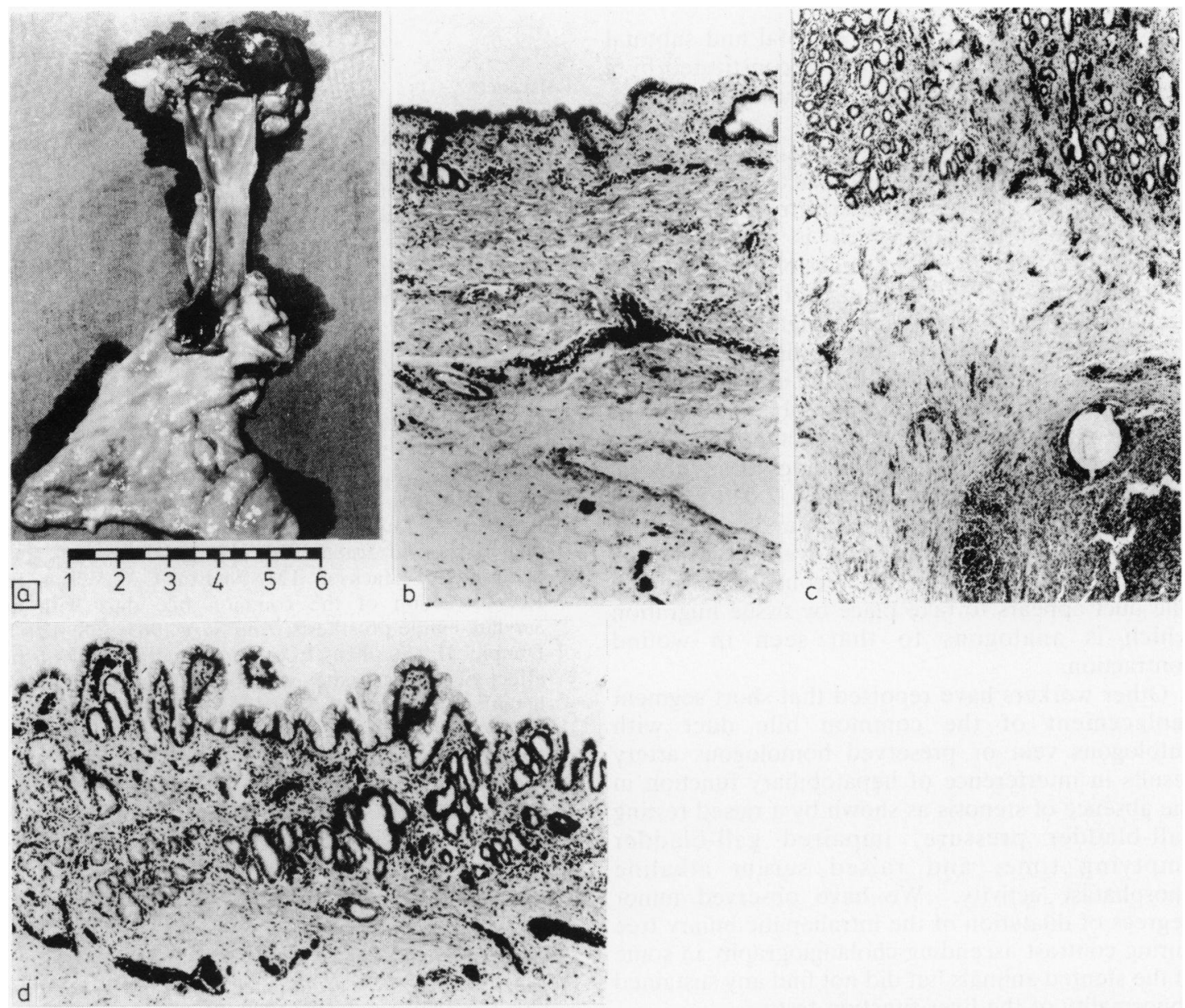

Fig. 3 (a) Close-up of the inner lining of common bile duct 12 months after total replacement with stenting showing single suture line scar at proximal end.

(b) Histology of duct above scar showing normal appearance.

(c) Histology at level of scar showing suture granuloma.

(d) Normal bile duct epithelium below suture line scar. 
Similar findings were observed in the group IV animals.

\section{RADIOLOGICAL STUDIES}

Cholangiography (Hypaque) studies were performed after cannulation of the bile duct through a duodenotomy at the time of death. Although no strictures were noted in the stented animals (groups II, IV), a minor degree of dilatation of the intrahepatic biliary tree was observed in three of these animals, but these animals had normal liver function tests although the liver biopsy at the time of death showed a polymorphonuclear infiltrate of the portal tracts.

\section{Discussion}

This study has shown that both total and subtotal excision of the bile duct can be reconstituted by a vein autograft provided biliary stenting is adopted, the absence of which invariably results in the development of dense strictures and extrahepatic bile duct obstruction. In the stented animals the vein appears to act as a temporary contracting scaffold, the venous intima of which is replaced with columnar epithelium within weeks of the operation. The longitudinal shortening of the graft is accompanied by progressive lengthening of the duodenal end of the bile duct. Ultimately the vein autograft disappears entirely and only one (proximal) suture lined is recognisable beyond four months of bile duct replacement by autologous vein. Belzer $e t a l^{8}$ reported similar findings after subtotal excision of the bile duct leaving a 2.0 to $3.0 \mathrm{~mm}$ posterior strip joining the two ends and replacement of defects with autologous vein over an indwelling T-tube. They concluded that reconstitution of the bile duct appears to take place by tissue migration which is analogous to that seen in wound contraction.

Other workers have reported that short segment replacement of the common bile duct with autologous vein or preserved homologous artery results in interference of hepatobiliary function in the absence of stenosis as shown by a raised resting gall-bladder pressure, impaired gall-bladder emptying time, and raised serum alkaline phosphatase activity. ${ }^{7}$ We have observed minor degrees of dilatation of the intrahepatic biliary tree during contrast ascending cholangiography in some of the stented animals but did not find any sustained abnormality of the liver function tests.
Grafting of bile duct strictures and defects in dogs with knitted Teflon results in incorporation of the interstices with collagen and an internal lining of bile duct epithelium which migrates from both sides of the prosthesis. ${ }^{459}$ In contrast to stented vein autografts, however, there is no shortening of the grafted segments.

At the present time there have been only a few clinical reports on common bile duct repair using grafting procedures. ${ }^{1011} \mathrm{~A}$ successful long term outcome of vein patch repair of common bile duct strictures, however, has been reported in two patients. In both instances the repair was stented for two and four months respectively.

\section{References}

1 Pearce AE, Ulin AW, Entine JH, Froio GF. Experimental reconstruction of the extrahepatic biliary system using free venous grafts. Ann Surg 1951; 134: 808-14.

2 Santos M, Smith ML, Hughes CW, Riley PA Jr. Reconstruction of the bile ducts. An experimental study using free arterial grafts and nylon mesh tubes. Surgery 1957; 42: 462-73.

3 Ulin AW, Van Ess L, Entine J, Pearce AE, Martin WL. Further experiences with the experimental reconstruction of the common bile duct: use of autogenous and homologous, fresh and preserved grafts of blood vessels, ureter and common duct. Am Surg 1953; 19: 867-3.

4 Myrin SO. The use of Teflon in reconstructive choledochal surgery. Acta Chir Scand 1960; 119: 118-20.

5 Hallberg O, Jonson G. Teflon choledochoplasty in dogs. Acta Chir Scand 1960; 119: 120-2.

6 Sherman RT, Jackson TM, Nielson CA, Wilson H. Reconstruction of the common bile duct with an acrylate-amide prosthesis. Ann Surg 1963; 158: 420-7.

7 Dunphy JE, Stephens FO. Experimental study of the effect of grafts in the common duct on biliary and hepatic function. Ann Surg 1962; 155: 906-19.

8 Belzer FO, Watts J.Mck, Ross HB, Dunphy JE. Auto-reconstruction of the common bile duct after venous patch graft. Ann Surg 1965; 162: 346-55.

9 Hartung $H$, Kirchner $R$, Baba N, Waldmann D, Strecker EP. Histological, laboratory and $\mathrm{x}$-ray findings after repair of the common bile duct with a teflon graft. Wld J Surg 1978; 2: 639-44.

10 Thomas JP, Metropol HJ, Myers RT. Teflon patch graft for reconstruction of the extrahepatic bile ducts. Ann Surg 1964; 160: 967-70.

11 Ellis $\mathrm{H}$, Hoile RW. Vein patch repair of the common bile duct. J Roy Soc Med 1980; 73: 635-7. 